\title{
Ueber den Gaswechsel der Tropenbewohner, speciell mit Bezug auf die Frage von der chemischen Wärmeregulirung.
}

\author{
Von \\ Dr. C. Eykman \\ aus Batavia.
}

Die Frage von der Regulirung der Körperwärme bei wechselnder Umgebungstemperatur ist in dieser Zeitschrift zu wiederbolten Malen Gegenstand eingehender Untersuchungen und Erörterungen gewesen. Namentlich bemilhten sich die betreffenden Forscher festzustellen, ob neben der Regulirung der Wärmeabgabe physikalische W.-R. im Sinne Rubner's - auch, wie schon Lavoisier annahm, eine Anpassung der Wärmeproduktion an die wechselnden Umgebungsbedingungen - che misch e W.-R. im Sinne Rubner's - stattfinde. Das Ergebniss dieser Forschungen war, insofern es sich um Thierversuche bandelte, nicht zweideutig; es lässt sich dabin zusammenfassen, dass in der Kälte eine gesteigerte Wärmeproduktion zu constatiren ist, als Folge eines vermehrten Stoffumsatzes, der als ein von dem Kältereiz ausgelöster Reflexvorgang aufufassen sei. Seitdem hat aber Löwy in diesem Arehiv (Bd. 46) gezeigt, dass jenes Resultat der Pfl üger'schen Schule nicht ohne weiteres auf den Menschen ausgedehnt werden darf. Hatte schon Voit sich dahin geäussert, dass unter den gemeiniglich beim Menschen obwaltenden Verhältnissen keine einigermaassen erhebliche Regulirung im erwähnten Sinne existire, und S p e ck eine solche sogar ganz in Abrede gestellt, so sah L öw y nur dann bei seinen Versuchspersonen einen vermebrten Sauerstoffverbrauch erfolgen, falls die Kälteeinwirkung unwillkürliche Muskelcontractionen hervorrief - unter Umständen also, die für die gewöhnlichen Verhältnisse als maassgebend nicht zu betrachten sind. Dazu waren die Zitterbewegungen nicht einmal im Stande das Sinken der Körpertemperatur aufuhalten, stellten somit auch im 
Verein mit der Hautwirksamkeit keine vollständige Regulirung dar. In allen denjenigen Fällen aber, wo von intelligenten, mit ibren Körperfunktionen vertrauten Individuen völlige Muskelschlaffheit angegeben wurde, war nie eine Zunahme des Sauerstoffverbrauchs zu constatiren.

Eine reflektorisch-chemische Wärmeregulation im P flügerschen Sinne hat mithin durch diese Versuche für den Menschen nicht nachgewiesen werden können. Indess ist festzuhalten, dass jene Experimente sich nur auf relativ kurz dauernde Temperatureinwirkungen beziehen. Wie ich aber in einer früheren Arbeit über den Stoffwechsel der Tropenbewohner hervorhob ${ }^{1}$ ), spricht eben eine bekannte Erfahrung dafür, dass eine fortd a u e r nde Ursache für vermehrte (bezw. verminderte) Wärmeverluste doch wohl eine Steigerung (bezw. Abnahme) der Wärmeproduktion nach sich ziehen kann. Es ist das die Thatsache, dass zwischen der Intensität des Stoffwechsels und der Entwickiung der Körperoberfläche ein inniger Zusammenhang besteht, in dem Sinne, dass kleinere Individuen mit ihrer relativ grösseren Körperoberfläche pro $\mathrm{kg}$ Körpergewicht mehr Wärme verlieren und demgemäss auch mehr produciren, als bei grösseren Individuen der Fall ist. Nach Analogie dieser Thatsache erscheint, auch rom Standpunkte der Physiologen, eine direkte Beeinflussung des Stoffwechsels bezw. der Wärmeproduktion durch die verschiedenen Jahreszeiten und Klimaten nicht ausgeschlossen. Gerade iiber diesen Punkt fehlte es jedoch an eingehenden Untersuchungen, wenn man wenigstens von einzelnen wenigen Thierversuchen absieht, die über den Einfluss der Jahreszeiten auf den Stoffwechsel angestellt wurden, ohne aber die betreffenden Autoren (Sen a tor, F in k ler, Herzog: Carl Theodor von Baiern) zur Einstimmigkeit bringen zu können. Was speciell das tropische Strandklima anbetrifft, sind solche Untersuchungen um so mehr angezeigt, als es hier nicht nur die hohe Temperatur, sondern auch die grosse Luftfeuchtigkeit ist, die die Wärmeabgabe behindert, und das Bedurfniss nach Herabsetzung der Wärmeproduktion doppelt in die Augen springen lässt.

Vor einigen Jahren habe ich in Virchow's Archiv (Bd. 133) berichtet ïber Stoffweehselversuche, die von mir bei in den Tropen

1) Virchow's Archiv, Bd. 133. 
lebenden Personen (Männern) ausgeführt waren. Dabei stellte sich heraus, dass der in Batavia lebende Europäer, von etwa $65 \mathrm{~kg}$ Körpergewicht, bei leichterer Arbeit täglich in der Nalrung aufnimmt :

\section{Eiweiss Fett C.-Hydrate Alkohol}

$99,6 \mathrm{gr} \quad 83,8 \mathrm{gr} \quad 264,2 \mathrm{gr} \quad 28,5 \mathrm{gr}$, wovon resorbirt werden: $88,2 \mathrm{gr} \quad 79,1 \mathrm{gr} \quad 256,4 \mathrm{gr} \quad 28,5 \mathrm{gr}$.

Nach Rubner ergiebt sich daraus ein Wärmewerth von brutto 2470, netto 2349 Cal. Nach der Immermann'schen Formel berechnet sich danach der Wärmewerth für einen $70 \mathrm{~kg}$ schweren Mann auf netto 2466 Cal.

Fïr annähernd die gleiche Arbeitskategorie wie die, wozu unsere Versuchspersonen gehörten, ist nach Rubner in Europa der einem $70 \mathrm{~kg}$ schweren Manne zukommende Wärmewerth durehschnittlich auf netto $2445 \mathrm{Cal}$. zu veranschlagen. Wir schlossen danach, dass sich eine regulatorische Herabsetzung der Wärmebildung bei dem, leichtere Arbeit leistenden, europäischen Tropenbewohner nicht nachweisen liess.

Ebensowenig liess sich aus den an unseren malayischen Versuchspersonen ermittelten Thatsachen auf das Bestehen einer chemischen Wärmeregulirung schliessen.

Unsere Ergebnisse bekräftigten somit die Ansicht, zu der Voit schon früher auf Grund ron theoretischen Ueberlegungen gelangt war und die er durch die allerdings noch spärlichen Nachrichten über die Kost in heissen Ländern bestätigt fand, dass nämlich für die höheren Wärmegrade keine in Betracht kommende Wärmeregulirung durch verminderte Oxydation zu existiren scheint.

Für diese Ansicht spricht auch die Erfahrung, dass die physikalische Wärmeregulation, die behufs einer genügenden Wärmeabgabe gesteigerte Hautfunktion, beim Tropenbewohner sich in hohem Maasse geltend macht. Dies wäre kaum verständlich, wenn eine einigermaassen beträchtliche Verminderung der Wärmeproduktion mit ins Spiel käme. Dass eine solche ganz ausgeschlossen sei, möchte ich dennoch bis auf Weiteres nicht behaupten und wäre das unter den gegebenen Versuchsbedingungen auch kaum zu beweisen. Man darf eben nicht aus den Augen lassen, dass die oben zum Vergleich aufgeführten Durchschnittszahlen für die Wärmeproduktion in Europa und in Indien keine absolut zuverlässigen Normen darstellen, sondern nur Annäherungswerthe sind, 
die von nebensächlichen Momenten, wie Unterschieden in der Lebensweise, den Ernährungsverbältnissen, der Beschäftigung u.s. w., sowie von individuellen Verschiedenheiten in Verbindung mit der beschränkten Zahl der Versuchspersonen, störend beeinflusst sein könnten.

Aus diesen Erwägungen heraus erschien es von Interesse, der Frage von der chemischen Wärmeregulirung beim menschlichen Tropenbewohner auch noch auf anderm Wege, und zwar durch die Untersuchung des respiratorischen Gaswechsels, näherzutreten.

In mancher Hinsicht entscheidende Resultate lassen sich, wie Zuntz c. s. bei verschiedenen Gelegenheiten erörtert haben, auch aus relativ kurz dauernden Respirationsversuchen gewinnen. Diese haben - abgesehen noch von ihrer leichteren Ausführbarkeit auch für uns den besonderen Vortheil, dass sich dabei, indem man nur ruhende und nüchterne Personen untersucht, wenigstens der direkte Einfluss der Ernährung und der Körperarbeit anf den Stoffumsatz ziemlich wohl ausschliessen lässt.

Was speciell die Beurtheilung der Wärmeproduktion anbetrifft, so ist festzuhalten, dass dafür die Bestimmung des Sauerstoffverbrauchs bei weitem melr maassgebend ist, als die der Koblensäureausscheidung. Denn, wie $\mathrm{R}_{\mathfrak{b}} b \mathrm{er}^{1}$ ) angiebt, wechselt die Calorienzahl, die bei der Produlstion einer bestimmten Kohlensäuremenge gebildet wird, im Verhältniss von 100:107:129, je nachdem Kohlenbydrat, Eiweiss oder Fett den Kohlenstoff dazu abgegeben haben. Für den Sauerstoffverbrauch sind die entsprechenden Verhältnisszahlen 100:84:92, wobei noch in Betracht zu ziehen ist, dass die Wärmeproduktion beim Menschen vorwiegend auf das Conto der Verbrennung von Kohlenhydrat und Fett kommt, für welche beiden Stoffe die entsprechenden Zahlen pro Aequivalent Kohlensäure gerade ziemlich viel (29 Proc.), pro Aequivalent Sauerstoff dagegen nur wenig ( 8 Proc.) auseinandergehen.

Der respiratorische Gaswechsel des Tropenbewohners wurde bis jetzt, sofern uns bekannt ist, noch nicht untersucht. Zwar begegnet man in der bezuglichen Literatur mitunter der Angabe, dass nach Rattray die Kohlensäureausscheidung in den Tropen nicht unerheblich herabgesetzt sei, beim Einsehen der Original-

1) Zeitschr. f. Biologie 21, s. 363. 
abbandlung ${ }^{1)}$ aber stellte sich heraus, dass dessen Angabe nicht auf thatsächlicher Beobachtung beruht, sondern lediglich das Ergebniss ist einer Berechnung, die von sehr anfechtbaren Prämissen ausgeht.

\section{Rat tray's Gedankengang ist folgender:}

Das Athemluftquantum ist, wie spirometrische Versuche lehren, im Tropenklima kleiner als im gemässigten Luftstrich. Die Differenz beträgt 7,567 Proc. für einen Temperaturunterschied von $18^{\circ} \mathrm{F}$. $\left(83-65^{\circ}\right.$ F. $)$, und dementsprechend wird auch die Kohlensäuremenge herabgesetzt sein, welche die Lungen nach aussen abzugeben im Stande sind. „But as tropic air contains less oxygen for a given bulk than air of colder latitudes, the decarbonating capabilities of the lungs will evidently be still further curtailed, and the amount of carbon they can throw off considerably decreased." Weil nun der Ausdehnungscoefficient der Gase pro Grad Fahrenheit $\frac{1}{480}$ beträgt, wird der erwähnte Unterschied:

$$
100-(100-7,567) \frac{480}{480+18}=10,9 \text { Proc. }
$$

In ähnlicher Weise begrïndet auch $J$ o as set seine $\Lambda$ nsicht „que la respiration est diminuée, qu'elle absorbe moins d'oxygène et qu'elle élimine moins d'acide carbonique" ${ }^{2}$ ).

Auffallend ist dabei jedoch, dass $R$ at $t r$ a y und $J$ ous s e t auf Grund von in geradem Widerspruch mit einander stehenden Annahmen dennoch zum gleichen Schluss kommen, dass nämlich die Lungenventilation im tropischen Klima weniger ergiebig ist. Ersterer fand, wie vor ihm andere englischen Forscher (D a v y Copland), die Athemfrequenz vermindert, die Menge der Respirationsluft hingegen ein wenig zugenommen :

\begin{tabular}{|c|c|c|c|c|}
\hline & $\begin{array}{l}\text { Cubic inche } \\
\text { in each } \\
\text { inspiration }\end{array}$ & & $\begin{array}{c}\text { Number } \\
\text { of } \\
\text { respirations }\end{array}$ & $\begin{array}{l}\text { Cubic inche } \\
\text { respired per } \\
\text { minute }\end{array}$ \\
\hline England & 15 & $x$ & 17 & $=255$ \\
\hline Tropies & 16,836 & $x$ & 14 & $=235,704$ \\
\hline
\end{tabular}

1) Proceedings Royal Society 1870.

2) Traité de l'acclimatement et de l'acclimatation, Paris 1884. 
Jous set constatirte demgegeniiber, in Uebereinstimmung: mit anderen französischen Beobachtern (Creva ux, Féris), dass die Athembewegungen in der heissen Zone an Frequenz zu- und an Tiefe abgenommen hatten:

in der gemässigten Zone $\ldots 16 \times 100=1600 \mathrm{ccm}$ pro Minute in der heissen Zone .....18 × $85=1530 \quad, \quad, \quad$, Differenz zu Gunsten der gemässigten Zone . . 70 ccm pro Minute oder 4,375 Proc.

Man ersieht, wie wenig begründet die Annahme ist, dass die Lungenventilation im tropischen Klima eine Verminderung erleidet. Aber auch wenn ihre Richtigkeit feststände, brauchte es kaum des Hinweises, dass eine Anschauung, wie jene der obengenannten Autoren, die den Stoffwechsel unmittelbar abhängig macht vom Gaswechsel in den Lungen, einen in der Playsiologie schon längst iiberwandenen Standpunkt kennzeichnet.

Es war La vo is i e r, der der Vorstellung Geltung verschaffte, dass kalte Luft, ihrer grösseren Dichtigkeit wegen, eine vermehrte Oxydation im Lungenblut veranlasst. L i e big, der anfänglich der gleichen Meinung war, ist später davon zurückgekommen, insofern als er die gesteigerte Sauerstoffaufnahme in den Lungen nicht mehr abhängig machte von der grösseren Dichtigkeit der kalten Luft, sondern von den in der Kälte energiseher vor sich gehenden Athem- und Herzbewegungen. Im übrigen beharite auch er auf der Meinung $L$ a $v$ o is i e r's, dass eine Mehraufnahme von Sanerstoff auch einen Mehrverbrauch bedinge ${ }^{1}$ ). Die Mehrheit der Autoren auf dem Gebiet der tropischen Medicin sind auf diesem Standpunkt stehen geblieben, während sie, was die Ursache der angeblich vermehrten Sanerstoffaufnahme anbetrifft, sowohl die Vorstellung von Lavoisier, als die von $L$ ie big acceptirten. Einige Citate ans Arbeiten der letzten Jahre diurften hier geniigen.

In seinem bekannten Buch "De geneesheer in Ned. Indie" (der Tropenarzt) giebt van den Burg die herrschende Anschanung, deren speculativen Charakter er freilich selbst hervorhebt, getreu wieder, indem er sagt: "Während die kalte Luft für sich schon die Athemmuskeln zu energischerer Wirkung anregt, miissen die Langen im heissen Klima jenen wohlthätigen Reiz entbehren.

1) Vergl. Voit, Hermann's Handbuch d. Physiologie, Bd. 6, Th. 1. 
Das unmittelbare Resultat der weniger tiefen Athembewegungen ist, dass das Blut weniger Sauerstoff aus der atmosphärischen Luft aufnimmt, welche letztere uberdies im gleichen Volum schon weniger Sauerstoff enthält, weil sie in Folge der Wärme stärker ausgedehnt ist, während Versuche ergeben haben, dass das Blut aus stark verdünnter Luft weniger Sauerstoff aufnimmt als aus dichterer Luft. Herabgesetzte Sauerstoffaufnahme hat mangelhafte Arteriellisirung des Blutes zufolge; das Blut wird im Ganzen von einer mehr venösen Beschaffenheit, es ist anoxyhämisch" ${ }^{1}$ ).

„Wahrscheinlich übernimmt die Leber zum Theil die den Lungen zukommende Funktion und verarbeitet sozusagen eine grössere Menge venösen Blutes zu den von ihr ausgeschieden werdenden Produkten".

Bei Orgeas (La pathologie des races humaines et le problème de la colonisation, 1886) lesen wir: „grâce à la diminution d'amplitude de la respiration et à la diminution probable du nombre absolu des mouvements respiratoires, la quantité d'air que reçoivent les poumons en vingt-quatre heures est considérablement diminuée dans les climats torrides. De plus, cet air, ayant une température de $25^{\circ}$, est moins riche en oxygène que l'air des climats tempérés, qui a une température moyenne de $12^{\circ}$. Il y a lieu de remarquer, en outre, que dans l'air chaud, l'oxygène a une tension moindre et, par suite, une moindre affinité pour les globules du sang (expériences de P. Bert). L'air que recoivent les poumons dans les climats torrides est done moindre comme quantité et comme qualité. Nous disons que l'air chauffé à $25^{\circ}$ est, à volume égal, moins riche en oxygène que l'air à la température de $12^{\circ}: 1^{0}$ paree que cet air, étant plus chaud, est plus dilaté; $2^{0}$ parce qu'il contient une quantité plus considérable de vapeur d'eau, qui prend la place d'une certaine quantité d'oxygène."

Auf den Einfluss der Luftfeuchtigkeit wird auch von Treille in seinem Werk: De l'acclimatation des Européens dans les pays chauds, Nachdruck gelegt: „Ce qui dans l'air des pays chauds diminue

1) Es muss eigenthümlich berühren, dass einst, gelegentlich einer von R. J. Mayer in Surabaya (Java) ausgeführten Venaesection, gerade die ge ringe Venösität des Aderblutes dessen Aufmerksamkeit erregte, eine Beobachtung, die für ihn der Ausgangspunkt war zu seiner Lehre von der Erhaltung der Kraft. 
l'absorption d'oxygène, c'est la tension de la vapeur d'ean qui, entrant dans la composition de la colonne barométrique, abaisse la tension propre de l'air sec, et la rend insuffisante" (S. 49). "Voilà la cause anémiante, la cause prohibitive d'une absorption d'oxygène adéquate aux besoins babituels de l'émigrant venu d'Europe. Joignons y l'effet nuisible de la chaleur, qui empêche l'oxygène, de se fixer avec énergie aux globules de sang" (S. 50).

Schon an anderer Stelle ${ }^{1}$ ) habe ich darauf hingewiesen, dass die Sauerstoffarmuth der feuchtwarmen Tropenluft schon darum von keinem besondern Einfluss sein kann auf die Sauerstoffaufnahme, weil es im kalten Klima ebensowenig die trockenkalte Aussenluft, sondern die in den Athemwegen nahezu bis zu Körpertemperatur erwärmte und mit Wasserdampf gesättigte Binnenluft ist, die sich direkt an dem Gaswechsel mit dem Lungenblut betheiligt.

Und auch, was das Abhängigkeitsverbältniss zwischen Sauerstoffaufnahme und Sanerstoffverbrauch anlangt, möchte ich nochmals hervorheben, dass es sich damit gerade umgekehrt verhält, als wie die oben citirten Autoren es vorstellen. Nach den neueren Ansichten in der Physiologie wird der Stoffumsatz nicht direkt von der Sauerstoffzufuhr beherrscht, sondern ist die Rolle des Sanerstoffs dabei vielmehr eine secundäre, indem derselbe nach den jedesmaligen Bedürfnissen des Organismus aufgenommen und verbraucht wird, und der Organismus über Compensationsmechanismen verfügt um, wo die Bedingungen für die Sauerstoffaufnahme ungïnstige sind, dennoch den gestellten Anforderungen, bis zu einem gewissen Maasse wenigstens, genügen zu können ${ }^{2}$ ).

Nach Obigem ist es einleuchtend, dass ein verminderter Sauerstoffverbrauch, gesetat dass derselbe in den Tropen vorhanden

1) Virchow's Archiv Bd. 131.

2) Diese Compensationsmittel sind so mächtig, "dass auch bei grosser Athemnoth, wenn sie längere Zeit ertragen wird, nicht weniger Sauerstoff in den Körper anfgenommen wird, und raseh der Tod unter Asphyxie eintritt sobald dies nicht mehr möglich ist." (Voit, a. a. O.)

Neuerdings haben $\mathrm{Gumberg}$ und $\mathrm{Zun} \mathrm{tz}$ den Sauerstoffverbrauch beim Aufenthalt in der verdünnten Bergluft sogar gesteigert gefunden. Dieses Archiv Bd. 63, S. 461. 
wäre, jedenfalls nicht ohne Weiteres sich aus der Sauerstoffarmuth der Atmosphäre erklären liesse.

"Die rein meteorologischen Verbältnisse," - sagt Stokvis in seinem Vortrag "Ueber vergleichende Rassenpathologie" S. 5 (X. internat. medicin. Congress) - „diejenigen also, welche in der Lufttemperatur, in der Luftfeuchtigkeit, in der Wasserdampfspannung, in den Luftströmen, in der Windrichtung, in der Besonnung u. s. w. ihren Ausdruck finden, baben, soweit sie den menschlichen Organismus beeinflussen können, nach meiner Ansicht nur die Bedeutung, dass sie Störungen der Wärmeregulirung hervorufen können, sind also thermische Momente. Wo es sich um tropische thermische Schädlichkeiten handelt, können wir selbst den atmosphärischen Druck vernachlässigen. Im natürlichen Lauf der Dinge hat nur Erniedrigung des Luftdrucks als ätiologisches Moment Bedeutung. Und Erniedrigung des atmosphärischen Drucks in tropischen Ländern - man denke an das Hochplateau Mexicos, den in Vorder-Indien gelegenen "Hillstations" Darjeeling und Chakatra, an unseren javanischen Sanatoria: Sindanglaja, Gadok I. s. w. - heisst Verschwinden der tropischen Temperatur. Die hochgelegenen Länder und Oerter in den Tropen sind eben keine tropischen Länder und Oerter mehr."

Für die Untersuchung des respiratorischen Gaswechsels bedienten wir uns des $\mathrm{Z} \mathfrak{u} \mathrm{n} \mathrm{t} \mathrm{z}-\mathrm{G}$ e p pert'schen Apparats in jener Gestaltung, wie derselbe, mit den im Lauf der Zeit daran angebrachten Verbesserungen, vor Kurzem in diesem Archiv (Bd. 55) von $\mathrm{Mag}$ a us-Lev y beschrieben wurde. Was die Einrichtung des Apparats und die Versuchsanordnung anbetrifft, sei sonit zunächst auf diese Beschreibung verwiesen, der wir noch Folgendes hinzuzufügen haben.

Mit dem vom Exspirationsventil nach der Gasuhr führenden Rohre wurde mittelst eines kurzen, weiten Seitenrohres eine dünne geschmeidige Schweineblase in Verbindnng gebracht. Auf einer festen Unterlage ruhend and bloss mit einem, um ein Scharnier drehbares Brettehen beschwert, wird die Blase bei jeder Exspiration aufgetrieben, um, mit durch die schwach aspirirende Wirkung der Gasuhr, danach wiederum zusammenzufallen. Das Durchstreiclen der Exspirationsluft durch die Gasuhr wird in dieser Weise auf längere Zeit vertheilt, was eine nicht unbedeutende Ver- 
minderung der Widerstände zufolge hat. Die auf- und niedergehende Bewegung des Brettchens bot uns zugleich die Gelegenheit, die Athembewegungen zu registriren. Die Fortbewegung des berussten Papiers liessen wir dabei nicht durch ein eigenes Räderwerk besorgen, sondern mittelst der drehenden Achse der Gasuhr selbst geschehen, sodass stets gleichen Verschiebungen des Papiers gleiche Mengen durchströmender Luft entsprachen. Dies bot den besonderen Vortheil, dass wir nur noch die Zeit zu markiren brauchten, um nachher abmessen zu können, wieviel Luft auf jeden Moment des Versuchs in der Zeiteinheit passirt war. Nur musste, weil man es hier nicht mit einem continuirlichen Luftstrom zu thun hatte, die Zeiteinheit nicht zu klein gewählt werden.

Solcherweise liess sich genau kontrolliren, ob die Versuchspersonen mehr oder weniger regelmässig geathmet hatten.

Einige Beispiele, deren Auswahl ganz dem Zufall überlassen wurde, dürften beweisen, dass die Regelmässigkeit der Athmung: bei unseren Versuchen iiberhaupt wenig zu wünschen übrig liess.

$10 \mathrm{~L}$. Luft correspondirten mit $36^{1} / 2 \mathrm{~mm}$ Abscisslänge (Verschiebung des berussten Papiers). Die Verschiebungen pro Minute sind nach diesem Maassstabe lierunter in L. Luft umgerechnet. Die so erhaltene Zahl repräsentirt somit das Athemvolum; nur dass die Volumina hier nicht auf die von trockner Luft von Normaldruck und -Temperatur reducirt sind.

\begin{tabular}{|c|c|c|c|c|c|c|c|c|c|c|c|c|}
\hline $\begin{array}{l}\text { Versuchs- } \\
\text { personen }\end{array}$ & & $\underset{ن}{\stackrel{\dot{g}}{\mid}}$ & $\begin{array}{l}\dot{\Xi} \\
\dot{z} \\
\dot{v}\end{array}$ & $\mid \begin{array}{l}\dot{3} \\
\infty \\
0\end{array}$ & $\mid \begin{array}{l}. \\
\dot{+}\end{array}$ & $\begin{array}{l}\dot{\Xi} \\
\therefore \\
\therefore\end{array}$ & $\mid \begin{array}{l}0 \\
0 \\
0\end{array}$ & $\mid \begin{array}{l}\stackrel{\dot{\Xi}}{z} \\
N\end{array}$ & $\mid \begin{array}{l}\underbrace{\circ} \\
\infty\end{array}$ & $\mid \begin{array}{l}. j \\
\dot{z}\end{array}$ & $\begin{array}{l}\dot{g} \\
\dot{7}\end{array}$ & 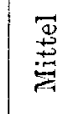 \\
\hline $\mathrm{N}$ & & & 7,2 & 7,0 & 7,8 & 7,7 & 8,0 & 7,4 & - & - & - & 7,4 \\
\hline 2. $\mathrm{Na}$. & $\begin{array}{l}\text { Resp } \\
\text { Res] }\end{array}$ & 28 & $\mid \begin{array}{r}8,2 \\
8,2 \\
18,5\end{array}$ & $\begin{array}{l}8,0 \\
8,2 \\
17\end{array}$ & $\mid \begin{array}{l}23 \\
7,5 \\
17\end{array}$ & $\begin{array}{r}8,8 \\
17\end{array}$ & $\left|\begin{array}{r}20 \\
8,5 \\
21,5\end{array}\right|$ & $\begin{array}{c}20,0 \\
7,7 \\
19\end{array}$ & $\begin{array}{c}\overline{7,1} \\
19\end{array}$ & $\overline{-}$ & $\overline{-}$ & $\begin{array}{l}22,1 \\
8,06 \\
18,6\end{array}$ \\
\hline 3. Fr. & & $\begin{array}{l}5,2 \\
18\end{array}$ & $\begin{array}{c}5,4 \\
18\end{array}$ & & $\begin{array}{c}4,7 \\
16\end{array}$ & $\begin{array}{r}5,2 \\
16,5\end{array}$ & 16 & $\left\{\begin{array}{c}6,4 \\
17\end{array}\right.$ & $\begin{array}{c}5,9 \\
18\end{array}$ & $\begin{array}{c}5,6 \\
17\end{array}$ & $\mid \begin{array}{c}6,1 \\
17\end{array}$ & $\begin{array}{c}5,47 \\
17,1\end{array}$ \\
\hline 4. Fl. & & $\begin{array}{r}6,2 \\
18,5\end{array}$ & $\begin{array}{c}6,3 \\
18\end{array}$ & $\begin{array}{r}6,6 \\
18,5\end{array}$ & $\begin{array}{r}9,6 \\
17,5\end{array}$ & $\left\{\begin{array}{c}6,6 \\
18\end{array}\right.$ & $\begin{array}{c}6,1 \\
17\end{array}$ & $\begin{array}{c}5,8 \\
17\end{array}$ & $\mid \begin{array}{c}5,9 \\
16^{6}\end{array}$ & $\begin{array}{l}6,3 \\
19\end{array}$ & $\mid \begin{array}{r}6,1 \\
17,5\end{array}$ & $\begin{array}{c}6,25 \\
17,7\end{array}$ \\
\hline 5. $\mathrm{Nr}^{*}$. & $\begin{array}{l}\text { Resp. } \\
\text { Resp }\end{array}$ & $\begin{array}{c}7,9 \\
19\end{array}$ & $\begin{array}{r}7,8 \\
19,5\end{array}$ & 18 & $\begin{array}{r}7,2 \\
18,5\end{array}$ & $\begin{array}{c}7,2 \\
18^{2}\end{array}$ & $\begin{array}{r}7,1 \\
18,5\end{array}$ & $\begin{array}{l}8,5 \\
19\end{array}$ & $18^{7,5}$ & - & - & $\begin{array}{c}7,59 \\
18,6\end{array}$ \\
\hline 6. K. d.J. & $\begin{array}{l}\text { Resp.-Vol. } \\
\text { Resp.-Freq. }\end{array}$ & $\begin{array}{c}5,8 \\
13\end{array}$ & $\begin{array}{r}6,2 \\
14,5\end{array}$ & 15 & 14 & $\begin{array}{c}7,0 \\
15\end{array}$ & $\mid \begin{array}{c}7,2 \\
15\end{array}$ & $\mid \begin{array}{r}7,4 \\
15,5\end{array}$ & $\begin{array}{r}7,7 \\
15,5\end{array}$ & $15^{7,4}$ & - & $\begin{array}{r}7,0 \\
14,7\end{array}$ \\
\hline
\end{tabular}

Die Abweichungen ron den Mittelwerthen lassen sich, wie folgt, gruppiren : 
Resp.-Vol. Resp.-Freq.

Abweichungen

$\begin{array}{ccrc}\text { von } 0-5 \text { Proc. } & 28 & 35 \\ 5-10 & 18 & 16 \\ " 10-17 & \Rightarrow & \frac{6}{52} & -\frac{1}{52}\end{array}$

Wie ersichtlich, gehören Abweichungen von mehr als 10 Proc. zu den Ausnahmen; meistentheils sind sie kleiner als 5 Proc.

Wir bemerken hierzu, dass der eigentliche Versuch, und damit die Registrirung der Athmung, stets erst angefangen wurde, nachdem die Versuchsperson schon einige Minuten durch den Apparat geathmet hatte. Denn in den ersten Minuten nach Be. ginn der Athmung am Apparat sind die Abweichungen rielfach bedeutender.

Im Uebrigen möchten wir nicht behaupten, dass die Athmung, so was Frequenz als Volum anbetrifft, bei derartigen Versuchen vollkommen mit der eines frei athmenden Individuums übereinstimme. Für uns, die wir speciell eine Parallele ziehen wollen zwischen dem respiratorischen Gaswechsel des Tropenbewohners and demjenigen des Bewolners kälterer Regionen, wird indess diese mögliche Fehlerquelle auf ein Minimum reducirt werden, wenn wir von den bezüglichen Untersuchungen nur diejenigen zum Vergleich heranziehen, die gleich wie die unsrigen mit dem Zuntz-Geppert'schen Apparat ausgefihhrt wurden. Gerade in dieser Beziehung bietet diese Zeitschrift wohl das umfangreichste Material dar und haben wir davon, sowie einer Abhandlung Ge ppert's im Archiv für experimentelle Pathologie, die in der umstehenden Tabelle gesammelten Daten entlehnt. Diese sind alle „Ruhe- und Nïchternwerthe“, d. h. sie beziehen sich auf Personen, die während des Versuchs nüchtern und in Ruhe waren. Auch wurden die betreffenden Versuche meistentheils in der kälteren und in keinem Falle in der heissen Jahreszeit angestellt. Dass unter diesen einschränkenden Bedingungen die Ausbeute an geeignetem Vergleichsmaterial noch zu wünschen übrig liess, braucht nicht Wunder zu nehmen. 


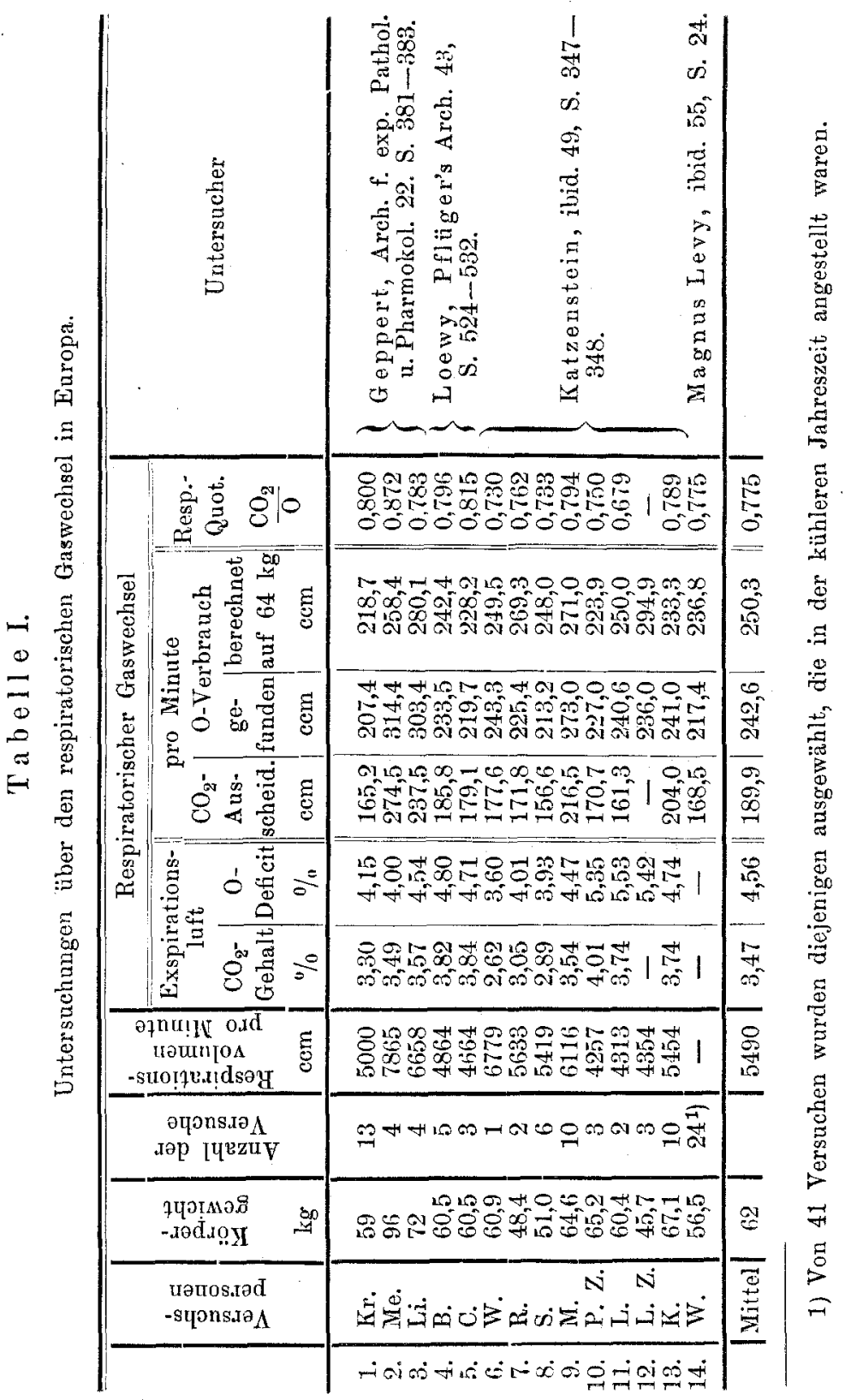


Um den Einfluss der verschiedenen Körpergrösse der Versuchspersonen auszugleichen, geben die citirten Untersucher auch die Kohlensäureabgabe und die Sanerstoffaufnahme pro kg Körpergewicht an. Ca eteris paribus aber ist die Grösse des Stoffwechsels entschieden nicht dem Körpergewicht, sondern vielmelır der Körperoberfläche proportional, sodass sie sich bei Personen von verschiedenem Gewicht $G$ und $g$ nicht verhält wie $G: g$, sondern etwa wie $\sqrt[3]{G^{2}}: \sqrt[3]{g^{2}}$ Dementsprechend haben wir mithin die Sauerstoffaufnahme aller Versuchspersonen, sowohl jener von Tab. I, als der unsrigen, anf ein gleiches Körpergewicht $(64 \mathrm{~kg})$ umgerechnet.

Der $\mathrm{CO}_{2}$-Gehalt der eingeatbmeten Luft haben wir nach dem Beispiel der Untersucher in Europa vernachlässigt; das Einathmungsventil befand sich bei unseren Versuchen in der Nähe eines geöffneten, in's Freie führenden Fensters.

Der Sauerstoffgehalt der atmosphärischen Luft wird von $\mathrm{Zuntz}$ c. s. bei ihren Versuchen auf 20,93 Proc. constant angenommen. Aus den Angaben von Magn us-Levy aber, der sich ïber diesen Punkt am ausführlichsten ausspricht, ergiebt sich, dass der nit der Hempel'schen Phosphorpipette ermittelte Sauerstoffgehalt zwischen 20,73 und 20,97 Proc. schwankte und im Durchschnitt 20,87 Proc. betrug. Er fuigt hinzu - wobei im Dunkeln bleibt, ob sich das auf die angefïhrten Zahlen bezieht - , ,dass bei den Controlluntersuchungen die Sauerstoffbestimmung gelegentlich grössere Abweiclungen ergab, ohne dass der Grund dafür mit Sicherheit nacbgewiesen werden konnte".

An unseren $\mathrm{Hem} \mathrm{pel'schen} \mathrm{Phosphorpipetten} \mathrm{machten} \mathrm{wir}$ die unliebsame Beobachtung, dass sie, aus unbekannter Ursache, frisch gefüllt den Sauerstoff ganz ungenügend absorbirten, sodass sehr niedrige Zahlen, bis zu 19,8 Proc., gefunden wurden. Erst im Verlauf von zwei Wochen etwa, näherten sich die ermittelten Zahlen allmählich der Norm, fielen aber immer noch etwas niedriger aus als bei Magnus-Levy; sie variirten von 20,65-20,88 Proc. Weil aber eine Differenz von 0,1 Proc. in der Bestimmung des Sauerstoffgebalts der Exspirationsluft schon einen Febler von 2-3 Proc. in der Bestimmung des Sauerstoffverbrauchs bedingt, so haben wir es für nöthig erachtet, uns dagegen za verwahren, indem wir bei allen Versuchen auch Controllanalysen der atmosphärischen Luft machten. Dadurch - und wir werden darauf zurückzukommen 
haben bei der Besprechung unserer Resultate - sind unsere Bestimmungen des Saterstoffverbrauchs etwa 3 Proc. niedriger, als wenn wir denselben einen constanten Sanerstoffgehalt der Atmosphäre von 20,97 Proc. zu Grunde gelegt bätten.

T abelle II.

Der respiratorische Gaswechsel in Indien.

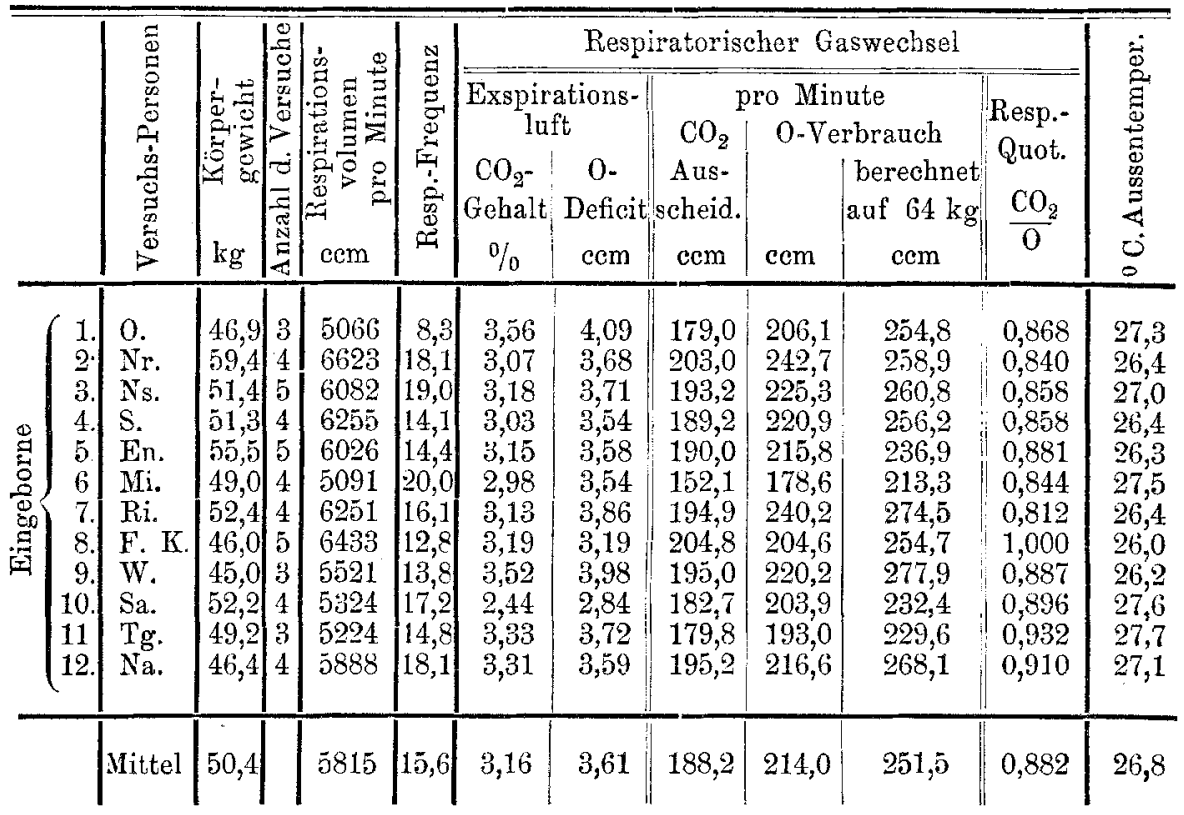

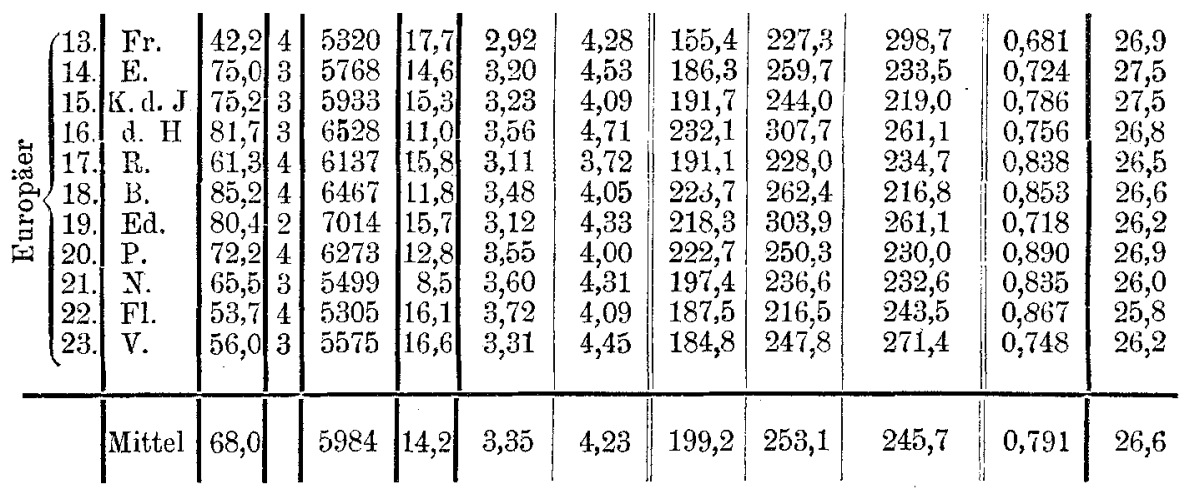

In obenstehender Tabelle sind die Endergebnisse unserer Versuche aufgeführt, die sämmtlich in Batavia angestellt sind. 
Untersucht wurden 12 Malayen und 11 Europäer, obne Ausnahme männliche erwachsene Personen, meistentheils im Alter von 20-40 Jabren. Einen einzigen ansgenommen ( $\mathrm{Nr}$. 20), der erst vor einigen Wochen in Indien angekommen war, batten die Europäer schon mehrere Jahre in den Tropen gelebt.

Die Malayen waren Anstalts- und Hansdiener, an leichtere Arbeit gewöhnt, mit meistentheils ziemlich gut, aber keinesfalls besonders stark entwickelter Muskulatur und mit nur mässig oder wenig entwickelter subcutaner Fettschicht.

Unter den Europäern befanden sich ein Laboratoriumdiener, ein Krankenwärter, ein Apotheker, drei Bureaubeamten und fünf Aerzte. Von ihnen war bloss einer (Nr.16) - ein Arzt, der täglich während einiger Stunden mit Massage beschäftigt war - wirklich stark muskulös. Die übrigen verrichteten keiner von Allen anstrengende Muskelarbeit; in dieser Hinsicht standen sie, im Durchschnitt genommen, ohne Frage noch hinter unseren malayischen Versuchspersonen zurück. Im Gegensatz zu diesen hatten viele Europäer einen gut, nicht selten sogar reichlich entwickelten Panniculus adiposus.

Es ist nöthig, diesen Besonderheiten Rechnung zu tragen, weil, aus naheliegenden Gründen, muskulöse Personen auch während der Körperruhe einen energischeren Stoffwechsel aufweisen als weniger kräftig gebanie, während andererseits Fettreichthum den Stoffumsatz herabsetzt.

Weiter unten, im Anhang, finden sich die übrigen Daten betreffs unserer Versuchspersonen und der an dieselben angestellten Versuche mitgetheilt. Um sich einigermaassen. eine Vorstellung machen za können von der Körpergestalt der betreffenden Personen, wurde daselbst, ausser dem Gewicht, auch die Körperlänge sammt dem Quotient dieser beiden $\left(\frac{G}{L}\right)$ angegeben.

Was zunächst den uns aus schon erwähnten Gründen am meisten interessirenden Sauerstoffverbrauch anbelangt, so bieten die volstehenden Tabellen nachfolgende, auf ein Körpergewicht von 64 $\mathrm{kg}$ berechnete Durchschnittsziffern zum Vergleich dar:

O-Verbauch pro Minute

Europäer in Europa (kühlere Jahreszeit) . . 250,3 ecm

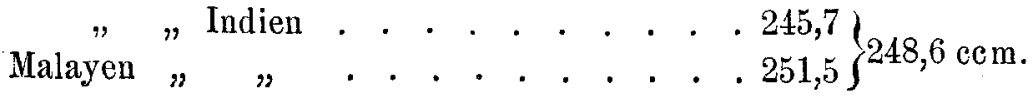


Wir constatiren folglich eine Uebereinstimmung zwischen dem Sauerstoffverbrauch in Europa und in Indien, wie sie genaner kaum zu erwarten sein dürfte. Die sich ergebenden Differenzen gehen nicht uiber die in unserem Falle ziemlich engbegrenzten Bestimmungsfehler hinaus und sind deshalb als unwesentlich zu betrachten.

Die Berechnung der Bestimmungsfehler nach der Methode der kl einsten $Q u a d r a t e$ ergiebt Folgendes:

Die ron uns für Europa angenommene Norm $\left(\mathrm{N}_{1}=250,3\right)$ ist mit cinem Fchler behaftet, dessen wahrscheinlicher Werth ist:

$$
\mathrm{F}_{1}= \pm 4,02 \text { (1,61 Proc.). }
$$

Unsere 23 Versuchspersonen ergaben eine Durchschnittszabl $\left(\mathrm{N}_{2}=248,6\right)$, dessen wahrscheinlicher Fehler:

$$
\mathrm{F}_{2}= \pm 2,50 \text { (1,01 Proc.). }
$$

Die Bestimmung der Differenz $\mathrm{N}_{1}-\mathrm{N}_{2}=1,7$ (oder 0,68 Proc.) ist mit einem Fehler behaftet, dessen wahrscheinlicher Werth noch etwas klciner ist als $\mathrm{F}_{1}+\mathrm{F}_{2}= \pm 6,52$ (2,6 Proc.).

Die kleinste Darchschnittszahl für den auf $64 \mathrm{~kg}$ Körpergewicht berechneten Sauerstoffgebranch weisen die europäischen Versuchspersonen in Indien anf. Freilich ist auch hier der Unterschied gegen Europa noch klein genug (1,8 Proc.; and ist demselben auch darum wohl keine Bedeutung beizumessen, weil an den Eingeborenen, bei denen sich eine Änassungserscheinung, wie die hier in Frage kommende, naturgemäss am ehesten kundgeben müsste, keine Abweichung im gleichen Sinne, wie bei dem europäischen Tropenbewohner $\mathrm{zu}$ constatiren ist. Auch ist festzuhalten, dass, wie oben hervorgehoben wurde, unsere Zablen bezïglich des Sauerstoffverbrauchs streng genommen um etwa 3 Proc. erhöht werden müssten, um völlig vergleichbar zu sein mit denen der Untersucher in Europa.

Von der anderen Seite dürfen wir nicht verschweigen, dass aus der eingangs erwähnten Arbeit von Löwy (dieses Archiv Bd. 46) Data zu entnehmen sind, die einen höheren mittleren Sauerstoffverbrauch ergeben, als der von uns für Europa angenommene, und zwar pro Minute und auf $64 \mathrm{~kg}$ Körpergewicht berechnet: $282,4 \mathrm{cem}$ anstatt $250,3 \mathrm{ccm}$, eine Differenz also von nabezu 13 Proc. Wir haben jedoch die betreffenden Data nicht in die Tabelle I aufgenommen, einmal weil einige der Versuchspersonen L ö wy's - es wird nicht angegeben, welche - nicht ganz nüchtern waren, und dann auch, weil Löw y's höherer 
Mittelwerth hauptsächlich auf das Conto zweier seiner Versuclıspersonen zu setzen ist, eines magern aber sehr muskulösen Tischlers (Hrm.) und eines kräftigen aber wenig fettreichen Schmieds (Ggld.), die - und das gilt auch von den meisten ubrigen - offenbar einer höheren Arbeitskategorie angehören, als die unsrigen. Fettarmuth aber und Muskulosität sind, wie schon bemerkt, Körpereigenschaften, die mit lebhafterem Stoffwechsel einhergehen. Sondern wir die beiden erwähnten Personen aus, so erhalten wir für sechs von Löwy's Versuchspersonen (Ulr., Strekm., Wnzl., Robrsch., Hdblg. und Lhm.) einen mittleren Sauerstoffverbrauch pro Minute und $64 \mathrm{~kg}$ Körpergewicht von $260,5 \mathrm{ccm}$, was nur um 4 Proc. höher ist, als der von uns für Europa angenommene Mittelwerth.

Folglich werden wir durch die Untersuchung des Sauerstoffverbrauchs zu demselben Schluss geführt, als wozu wir früher dureh die Untersuchung der Ernährungsverhältnisse gekommen waren, dass bei den menschlichen Tropenbewohnern keine einigermaassen in Betracht kommende chemische Wärmeregulationstattfindet.

Der Respirationsquotient ist, wenn wir die oben besprochene Correctur von +3 Proc. für den Sauerstoffverbrauch vornehmen, bei unseren enropäischen Versuchspersonen $\frac{0,791}{1,03}=0,768$, bei den Malayen $\frac{0,882}{1,03}=0,856$. Bei den Europäern der Tabelle I ist R.-Q $=0,775$. Die höhere Ziffer der Malayen erklärt sich ungezwungen aus ihrer kohlenhydratreicheren Nahrung. Dadurch anch (vgl. S. 60) repräsentirt jedes cem verbrauchten Sanerstoffs bei ihnen durchschnittlich eine etwas höhere Wärmeproduktion als bei den Europäern. Die relative, d. h. auf ein gleiches Körpergewicht berechnete Wärmeproduktion der Eingeborenen muss also diejenige der Europäer noch um etwas mehr übersteigen, als ihrem, übrigens sehr wenig höheren relativen Sauerstoffverbrauch entspricht. Dass in Wirklichkeit solch ein Unterschied bestehe, dass es nicht ganz vom Zufall abhängig, sondern vielleicht sogar grösser sein muss, als wir es fanden, kommt uns nicht unwahrscheinlich vor, angesichts des Umstandes, dass die malayischen Versuchspersonen meistentheils ziemlich muskulös waren, keiner von allen dagegen einen reichlich entwickelten Panniculus adiposus besassen, wie viele unserer Europäer. 


\section{Anhang.}

Versuchsprotokolle.

\begin{tabular}{|c|c|c|c|c|c|c|c|c|c|c|}
\hline Versuchspersonen & $\begin{array}{r}\text { Versuc } \\
\text { zeit }\end{array}$ & 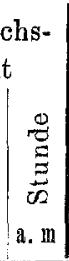 & 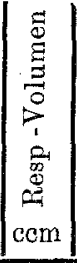 & 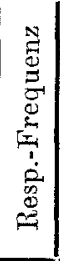 & $\begin{array}{l}\text { Res } \\
\text { Exsp } \\
\text { tions } \\
80 \\
80\end{array}$ & $\begin{array}{l}\text { spirat } \\
\text { pira- } \\
\text { sluft } \\
.2 \\
0 \\
0 \\
0 \\
0 \\
0\end{array}$ & $\begin{array}{r}\text { pro } \\
n \\
0 \\
0 \\
0 \\
0\end{array}$ & 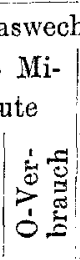 & 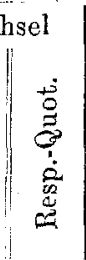 & 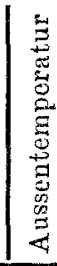 \\
\hline \multirow[t]{2}{*}{$\begin{array}{l}\text { 1. Eingeborener } 0 ., \\
\text { ca. } 40 \mathrm{~J} \text { alt, fettarm } \\
\text { und mässig muskulös. } \\
\mathbf{G .}=46,9 \mathrm{~kg}\end{array}$} & $\begin{array}{ll}20 . & 6 . \\
6 . & 7 .\end{array}$ & $\begin{array}{l}8 \\
8,30 \\
8,30\end{array}$ & $\begin{array}{l}5008 \\
5199 \\
4990\end{array}$ & $\frac{-}{8,3}$ & $\mid \begin{array}{l}3,34 \\
3,51 \\
3,82\end{array}$ & $\begin{array}{l}4,05 \\
4,02 \\
4,20\end{array}$ & $\begin{array}{l}164,4 \\
182,0 \\
190,6\end{array}$ & $\mid \begin{array}{l}199,2 \\
309,4 \\
209,6\end{array}$ & $\left\{\begin{array}{l}0,825 \\
0,870 \\
0,910\end{array}\right.$ & $\begin{array}{l}28 \\
28 \\
26\end{array}$ \\
\hline & $\mathrm{Mit}$ & & $\mid 5066$ & 8,3 & $|3,56|$ & $|4,09|$ & $\| 17$ & & & \\
\hline
\end{tabular}

II. 43 jähriger Euro-|30. 11.8,30|5736|17,0| 2,90|3,99 $166,4|228,7| 0,727 \mid 27$ päer Fr., Laboratoriumdiener, klein und sehr mager.

$$
\mathrm{G}=\frac{42,2}{16,2}=2,60
$$

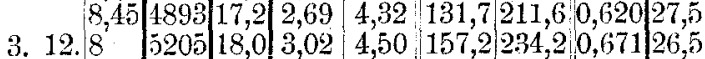
\begin{tabular}{l|l|l|l|l|l|l|l|l|l|l|l|l|l}
8 & 5205 & 18,0 & 3,02 & 4,50 & 157,2 & 234,2 & 0,671 & 26,5 \\
8,20 & 5448 & 18,5 & 3,05 & 4,31 & 166,2 & 234,8 & 0,708 & 26,5
\end{tabular}

Mittel $|5320| 17,7|2,92| 4,28|155,4| 2,27,3,0,681 \mid 26,9$

III. Eingeborener Nr., |10. 7. $|7,30| 6130|15,8| 3,19|4,30||195,6| 263,6|0,742| 25,5$ 25 Jahre alt, ziemlich muskulös, mässig fettreich.

$$
\frac{\mathrm{G}}{\mathrm{L}}=\frac{59,4}{16,9}=3,56
$$
$8,156744 \quad 19,0 \quad 3,16 \quad 3,50: 213,2236,50,903 \mid 26$ 5. 8. $7,45|6955| 19,3 \quad 2,97 \quad 3,53 \mid 206,5245,50,84126$ \begin{tabular}{l|l|l|l|l|l|l|l|l|l|l|l|l|l|l|l|l|l|l|} 
15. 10.8 & 6665 & 18,4 & 2,95 & 3,38 & 196,6 & 225,3 & 0,873 & 28
\end{tabular}

IV. Europäer E., $\mid$ 2: $1 .|9 \quad| 5391|14,9| 3,15|4,73| 169,8|255,000,666| 27$

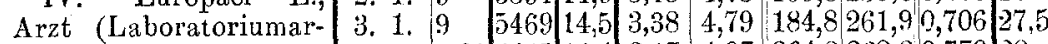
beit.), 37 Jahre alt, mässig fettreich u.muskulös.

$$
\frac{\mathrm{G}}{\mathrm{L}}=\frac{75,0}{18,5}=4,05
$$

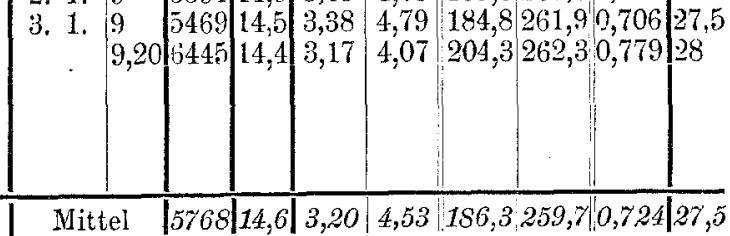

V. K.d.J., Europäer, $[23$. 6. $|8,45| 6220|16,4| 3,35|3,97| 208,4|246,90,844| 28$

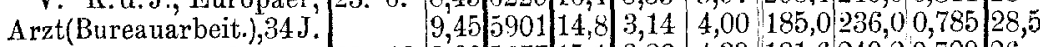
alt, fettreich und ziem- 22. 12.8,30 $5677|15,1| 3,20$ 4,39 $181,6|249,20,729| 26$ lich muskulös.

$$
\mathrm{G}=\frac{75,2}{16,4}=4,59
$$




\begin{tabular}{|c|c|c|c|c|c|c|c|c|c|c|}
\hline Versuchspersonen & $\begin{array}{r}\text { Versu } \\
\text { zei } \\
\stackrel{\substack{\infty \\
=}}{c}\end{array}$ & 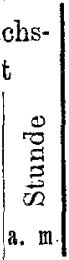 & 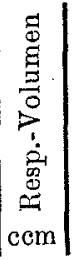 & 造 & 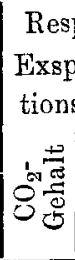 & 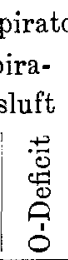 & 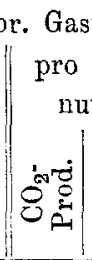 & 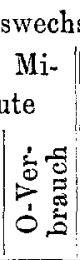 & 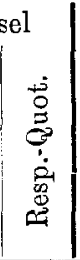 & 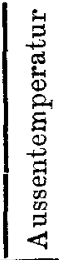 \\
\hline $\begin{array}{l}\text { VI. 34 jähriger Furo- } \\
\text { päer d. H., Arzt (Mas- } \\
\text { seur), muskulös und } \\
\text { ziemlich fettreich. } \\
\quad \overline{\mathrm{G}}=\frac{81,7}{17,6}=4,64 \\
\mathrm{~L}\end{array}$ & $\begin{array}{r}25.6 . \\
17.7 . \\
8.8 .\end{array}$ & $\begin{array}{l}9 \\
9,15 \\
9\end{array}$ & $\begin{array}{l}6476 \\
6790 \\
6320\end{array}$ & $\begin{array}{l}\overline{10,5} \\
11,5\end{array}$ & $\begin{array}{l}3,42 \\
3,60 \\
3,65\end{array}$ & $\begin{array}{l}4,48 \\
4,90 \\
4,75\end{array}$ & $\begin{array}{l}221,4 \\
244,4 \\
230,6\end{array}$ & $\begin{array}{l}290,1 \\
332,7 \\
300,2\end{array}$ & $\begin{array}{l}0,764 \\
0,735 \\
0,768\end{array}$ & $\begin{array}{l}26,5 \\
27 \\
27\end{array}$ \\
\hline
\end{tabular}

VII. Europäer R., 26. 6. $|7,30| 5607|13,2| 3,03|3,68| 169,9|206,3| 0,823 \mid 27$ Arzt (Laboratoriumarbeit.), 29 Jahre alt, ziemlich fettreich u.muskulös

$$
\frac{\mathrm{G}}{\mathrm{L}}=\frac{61,3}{16,5}=3,72
$$
\begin{tabular}{ll|l|l|l|l|l|l|l|l|l|l|l|l|l|}
\hline 8,30 & $\mathbf{b} 199$ & 15,2 & 3,03 & 3,77 & 187,7 & 233,3 & 0,804 & 27
\end{tabular}

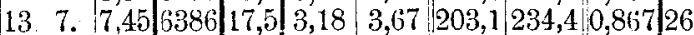
$\left[\begin{array}{l|l|l|l|l|l|l|l|l|l|l|}\hline 8,15 & 6358 & 17,3 & 3,20 & 3,74 & 203,5 & 237,8 & 0,856 & 26\end{array}\right.$

Mittel $|6137| 15,8|3,11| 3,72|191,1| 228,0,0,838 \mid 26,5$

VIII. 31 jähriger Eu- [11. 7. 7 [5867|11,0| $3,87|4,46| 227,0|261,60,865| 26,5$ ropäer, B., Apotheker, 17. 7. 7,30 7155 $13,0 \mid 3,14$ 3,96 $224,7288,30,79326$

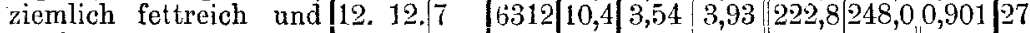
muskulös.

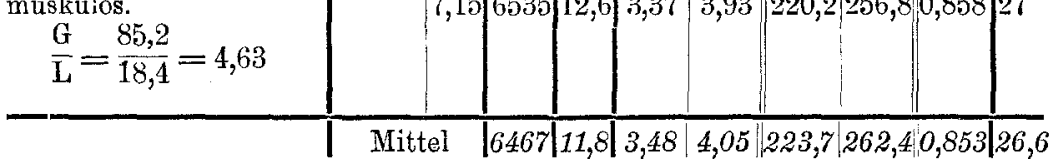

IX. Eingeborener Ns., ||2. 7. $|8,15| 6250|21,7| 2,70|3,14| 168,8|196,3| 0,860 \mid 26$

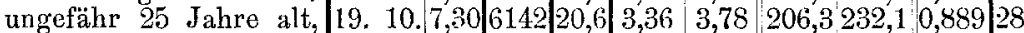
mässig fettreich, ziemlich muskulös.

$$
\frac{\mathrm{G}}{\mathrm{L}}=\frac{51,4}{15,9}=3,23
$$$$
\begin{array}{l|l|l|l|l|l|l|l|l|l|l|l|l|}
\hline 8 & 6323 & 20,1 & 3,38 & 3,65 & 213,7 & 232,7 & 0,918 & 28
\end{array}
$$

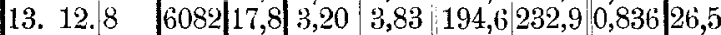
\begin{tabular}{l|l|l|l|l|l|l|l|l|l|l|l|l|l|l|l|}
8,15 & 5612 & 14,7 & 3,25 & 4,14 & 182,4 & 232,4 & 0,785 & 26,5
\end{tabular}

Mittel $|6082| 19,0|3,18| 3,71|| 193,2|225,3| 0,858 \mid 27$

X. Eingeborener S., $|15.7| 7,.45|6236|-|3,32| 3,93|207,0| 245,1|0,845| 26$ $\pm 35 \mathrm{~J}$. alt, fettarm und muskulös.

$$
\frac{\mathrm{G}}{\mathrm{L}}=\frac{51,3}{16,7}=3,07
$$




\begin{tabular}{|c|c|c|c|c|c|c|c|c|c|}
\hline Versuchspersonen & 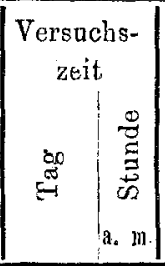 & 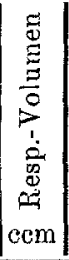 & 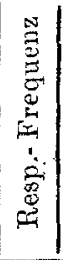 & 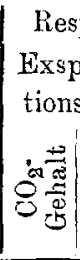 & $\begin{array}{l}\text { pirato } \\
\text { aira- } \\
\text { sluft } \\
\qquad \\
0 \\
0 \\
0 \\
0 \\
0\end{array}$ & 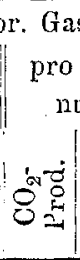 & 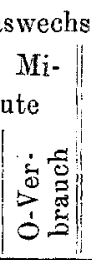 & 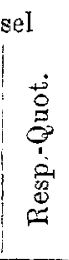 & 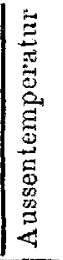 \\
\hline $\begin{array}{l}\text { XI. Bejahrter Malaye, } \\
\text { En., mässig fottreich } \\
\text { und muskulös. } \\
\qquad \mathrm{G}=\frac{55,5}{16,5}=3,36\end{array}$ & \begin{tabular}{ll|l|}
16. & 7. & 7,45 \\
8,30 \\
29. & 10. \\
8 \\
8,15 \\
$8,16$. & $12 . \mid$ \\
8
\end{tabular} & \begin{tabular}{|l|}
6650 \\
6659 \\
5570 \\
5591 \\
5659
\end{tabular} & \begin{tabular}{|l|}
14,3 \\
15,5 \\
14,2 \\
14,4 \\
13
\end{tabular} & $\begin{array}{l}3,32 \\
3,21 \\
2,84 \\
2,82 \\
3,55\end{array}$ & $\mid \begin{array}{l}3,71 \\
3,60 \\
3,48 \\
3,33 \\
3,76\end{array}$ & \begin{tabular}{|l|}
220,8 \\
213,7 \\
158,1 \\
156,5 \\
200,9
\end{tabular} & $\begin{array}{l}246,70 \\
239,70 \\
193,80 \\
186,10 \\
212,80\end{array}$ & $\begin{array}{l}0,895 \\
0,892 \\
0,816 \\
0,847\end{array}$ & $\begin{array}{l}26 \\
26,5 \\
26 \\
26 \\
27\end{array}$ \\
\hline & & & & 17 & & & & & \\
\hline
\end{tabular}

XII. Mi., 25 jähriger $\mid$ 18. 10.7,30|4260|20,4 $|2,80| 3,64|119,3| 155,10,770 \mid 28$

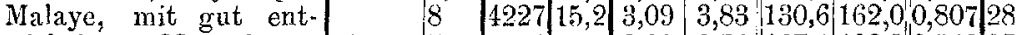
wickelter Muskulatur, 13. 12.7,30|5796 22,7 2,90 3,34 167,1,193,50,86827 wenig fettreich.

$$
\stackrel{\mathrm{G}}{\mathrm{L}}=\frac{49,0}{15,6}=3,14
$$

| Mittel $|5091| 20,0|2,98| 3,54,152,1|178,6,0,844| 27,5$

XIII. Ri., etwa $30|18.7| 7,.45|5678| 12,8|3,43| 4,34|194,7| 246,4|0,791| 26.5$ jähriger Malaye, mässig fettreich u. muskulös.

$$
\stackrel{G}{L}=\frac{52,4}{16,6}=3,16
$$

XIV. Eingeborener $19.7 . \quad 7,30|6010| 13,2|3,21| 3,61|192,9| 217,0|0,881| 26$ T. K., ca. 30 J.alt, fett- 31 . 7. $7,30684515,1 \quad 3,16$ 3,13 $216,3214,21,010$ arm, mässig muskulös.

$$
\begin{aligned}
& \mathrm{G} \\
& \mathrm{L}^{-}
\end{aligned}=\frac{46,0}{15,8}=2,91
$$$$
7,45[5747-2,942,81 \mid 198,3190,91,03526
$$

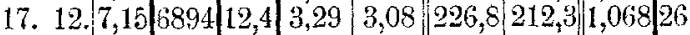$$
\begin{array}{|l|l|l|l|l|l|l|l|l|l|l|}
7,3,30 & 5671 & 10,5 & 3,35 & 3,33 & 189,9 & 188,8 & 1,006 & 26
\end{array}
$$$$
\text { Mittel |6433|12,8|3,19|3,19|204,8:204,61,000|26 }
$$

XV. 35 jähr. Euro- 24.7 . $|7,30|$; $928|13,7| 3,28|4,52:| 227,2|313,10,726| 26,5$

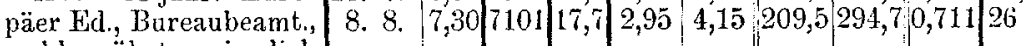
wohlgenährtes, ziemlich kräftiges Individuum.

$$
\dot{\mathrm{L}}=\frac{80,4}{17,9}=4,49
$$

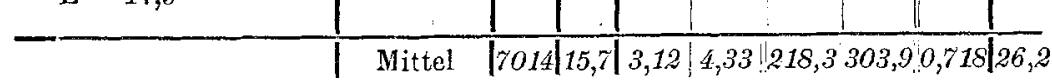

XVI. W., 30 jähr. Ma- $1.8 .18 \quad 5336|14,9| 3,29|3,64| 175,5|194,2| 0,904 \mid 26$

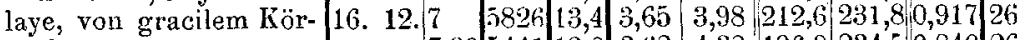
perbau.

$$
\frac{\mathrm{G}}{\mathrm{L}}=\frac{45,0}{16,2}=2,78
$$




\begin{tabular}{|c|c|c|c|c|c|c|c|c|c|c|}
\hline Versuchspersonen & $\begin{array}{r}\text { Versu } \\
\text { zei }\end{array}$ & 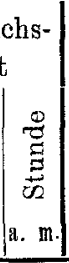 & 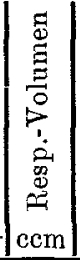 & 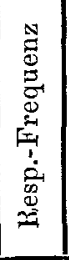 & \multicolumn{4}{|c|}{\begin{tabular}{|l||}
\multicolumn{2}{|c|}{ Respirator. Gaswechs } \\
Exspira- \\
tionsluft
\end{tabular}} & 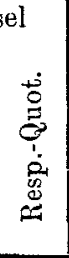 & 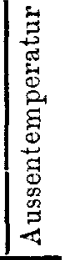 \\
\hline $\begin{array}{l}\text { XVII. Sa., Malaye, et- } \\
\text { wa } 25 \text { Jahre alt, wenig } \\
\text { fet.treich, mässig ent- } \\
\text { wickelte Muskulatur. } \\
\qquad \frac{\mathrm{G}}{\mathrm{L}}=\frac{52,2}{16,3}=3,20\end{array}$ & $\begin{array}{ll}12 . & 10 \\
16 . & 10 \\
22 . & 10\end{array}$ & $\begin{array}{l}8 \\
8,15 \\
7,30 \\
7,45\end{array}$ & $\begin{array}{l}5510 \\
5140 \\
5140 \\
5345 \\
5300\end{array}$ & $\begin{array}{l}16,8 \\
16,4 \\
18,1 \\
17,3\end{array}$ & $\begin{array}{l}2,97 \\
3,47 \\
3,61 \\
3,70\end{array}$ & $\begin{array}{l}3,31 \\
3,80 \\
4,05 \\
4,18\end{array}$ & $\begin{array}{l}163,6 \\
178,4 \\
192,9 \\
196,1\end{array}$ & $\mid \begin{array}{l}182,4 \\
195,3 \\
216,4 \\
221,5\end{array}$ & $\left|\begin{array}{l}0,897 \\
0,913 \\
0,891 \\
0,885\end{array}\right|$ & $\begin{array}{l}28 \\
28,5 \\
27 \\
27\end{array}$ \\
\hline & & & 89 & & & & & & & \\
\hline
\end{tabular}

XVIII. Eingeborener |16. 10.|7,30|ว5536|14,4|3,37|3,47||186,6,192,1 $0,971 \mid 28$ Tg., 25 J. alt, mittelkräftig und -fettreich.

$$
\frac{\mathrm{G}}{\mathrm{L}}=\frac{49,2}{15,5}=3,18
$$

XIX. 20 jähriger Ma- $9.12 .8 \quad[5118|18,7| 3,12|3,54||159,7| 181,1|0,822| 27,5$ laye Na. Pauniculus adiposus unid Muskulatur mässig entwickelt.

$$
\frac{\mathrm{G}}{\mathrm{L}}=\frac{4,64}{1,62}=2,87
$$

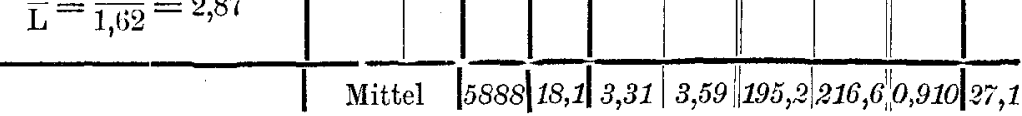

XX. 22 jähriger Euro- $|10.12| 7,.15|5852| 12,5|3,75| 4,41|219,5| 258,1|0,850| 27$

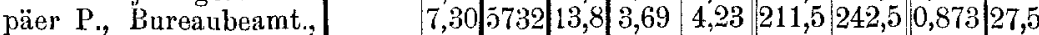

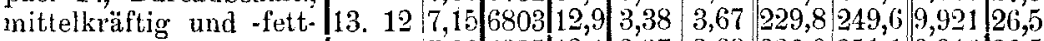
reich.

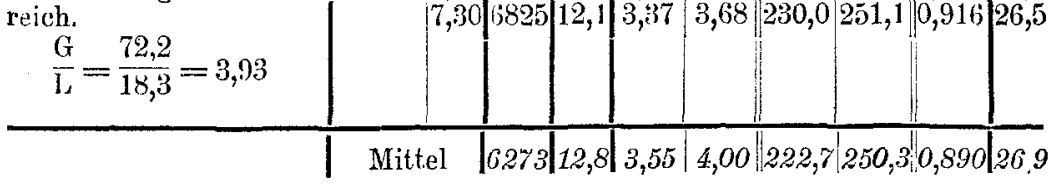

XX1. N., 30 jähriger |11. $12.7 \quad 5248|9,2| 3,87|4,60| 203,0|241,4| 0,841 \mid 26,5$

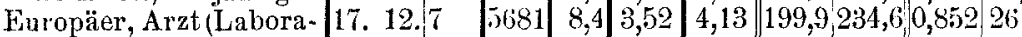

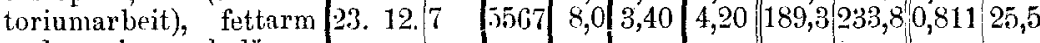
und wenig muskulös. $\frac{\mathrm{G}}{\mathrm{L}}=\frac{72,2}{18,3}=3,93$ 


\begin{tabular}{|c|c|c|c|c|c|c|c|c|c|}
\hline Versuchspersonen & 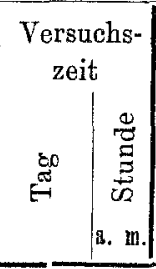 & 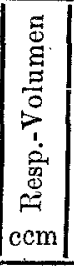 & 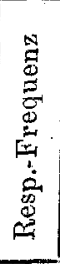 & \multicolumn{4}{|c|}{$\begin{array}{l}\text { Respirator. Gaswechse } \\
\text { Exspira- } \\
\text { tionsluft }\end{array}$} & 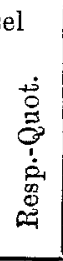 & 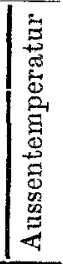 \\
\hline $\begin{array}{l}\text { XXII. } 40 \text { jülhriger Eu- } \\
\text { ropäer Fl, Krankenwär- } \\
\text { ter, klein und mager. } \\
\qquad \frac{\mathrm{G}}{\mathrm{L}}=\frac{53,7}{16,6}=3,23\end{array}$ & \begin{tabular}{|ll|l|}
27. & $12 \cdot$ \\
3. & 1. & 7,15 \\
7,15
\end{tabular} \mid & $\begin{array}{l}5500 \\
5326 \\
5410 \\
1984\end{array}$ & $\begin{array}{l}17,2 \\
17,4 \\
15,0 \\
13,8\end{array}$ & $\begin{array}{l}3,26 \\
3,34 \\
3,67 \\
3,90\end{array}$ & \begin{tabular}{l|}
3,95 \\
4,15 \\
3,87 \\
4,38
\end{tabular} & $\begin{array}{l}179,3 \\
177,9 \\
198,5 \\
194,4\end{array}$ & $\mid \begin{array}{l}267,3 \\
221,0 \\
209,4 \\
218,3\end{array}$ & $\begin{array}{l}0,825 \\
0,805 \\
0,948 \\
0,890\end{array}$ & $\mid \begin{array}{l}25,5 \\
25,5 \\
26 \\
26\end{array}$ \\
\hline & & & & 72 & & & & & \\
\hline
\end{tabular}

XXIJI. V., Europäer, 6. 1. $71 / 2|2539| 16,6|3,24| 4,87|173,0| 260,000,666 \mid 26,5$ $29 \mathrm{~J}$. alt, Bureaubeamt., 19. 1. 8 5691 - 3,21 4,05 182,7 $230,50,79326^{\circ}$

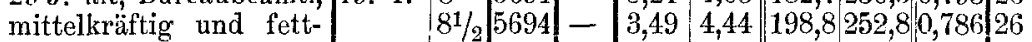
arm.

$$
\frac{\mathrm{G}}{\mathrm{L}}=\frac{56,0}{16,4}=3,42
$$

